\title{
Survey of oral anticoagulant treatment in children
}

D I K Evans, M Rowlands, L Poller

\begin{abstract}
Aims: To find out which children are treated with oral anticoagulants and how their treatment is controlled in the United Kingdom.

Methods: Two questionnaires were used. The first was sent to general haematologists and the other to paediatric cardiologists and cardiac surgeons.

Results: There were 273 (58\%) replies to the first questionnaire. Most children were treated because of artificial cardiac valve replacement. The mean target International Normalised Ratio (INR) used was 2.73 to 4.0 for children with heart valves and $2 \cdot 1$ to 3.25 for children with venous thrombosis. The second questionnaire elicited replies from 11 of 22 cardiac centres. The mean target INR used for children with cardiac valves ranged from 2.59-3.77. Of 68 children covered in the survey, there have been two major bleeds and two thrombotic episodes: $\mathbf{7 8 . 8 \%}$ of children were controlled with a venous prothrombin time and $21 \cdot 2 \%$ with a capillary test. There was no consistency in the dose regimens used for the induction of oral anticoagulant treatment with warfarin.

Conclusions: The levels of anticoagulation used for maintenance are similar to those recommended by the British Society for Haematology for adults $(3 \cdot 0$ to $4 \cdot 5)$. They seem to be safe for children too.
\end{abstract}

There are well documented guidelines for the control of oral anticoagulants in adults ${ }^{1}$; but oral anticoagulation of children is much less common. Little or no information is available on the use and particular problems associated with this treatment in childhood. We therefore undertook a survey of anticoagulant practice conducted by two groups of clinicians-haematologists and cardiologists or cardiac surgeons-involved in the initiation and control of oral anticoagulant treatment in children.

\section{Methods}

As a first step a questionnaire covering indications for use, optimal therapeutic ranges, and problems with warfarin treatment in children (up to age 14 years) was sent to 407 haematologists in Great Britain. Following analysis of their replies it became apparent that the largest group of children requiring oral anticoagulation were those with prosthetic cardiac valves. Therefore, a second modified questionnaire was sent to 77 consultants in 22 different centres specialising in paediatric cardiology and cardiothoracic surgery.

Copies of the questionnaire are available from the authors on request.

\section{Results}

QUESTIONNAIRE 1

Two hundred and thirty five out of 407 haematologists returned completed questionnaires. Of these, $173(73.6 \%)$ had no record of children attending their anticoagulant clinics. The remaining $62(26.4 \%)$ had controlled the warfarin treatment of 137 children during the previous five years. The age distribution and indications for anticoagulation of these patients are shown in tables 1 and 2 .

In each age group a prosthetic cardiac valve was the most common indication for oral anticoagulation-that is, six of eight (75\%) aged less than 2 years, 59 of $74(79 \cdot 7 \%) 2-10$ years, 36 of $55(65 \cdot 5 \%) 10-14$ years.

A summary of the stated target therapeutic ranges for oral anticoagulation in children with prosthetic heart valves and venous thrombosis (all causes) is shown in table 3. The intensities of anticoagulation are expressed as International Normalised Ratios (INR).

A further five haematologists indicated that their optimal level of anticoagulation in children with prosthetic heart valves was "the adult range."

In children under 2 years of age there were no stated differences in the desired degree of anticoagulation, for any disease group.

Three haematologists reported some difficulty with anticoagulant control. Two of these

Table 1 Age distribution of patients

\begin{tabular}{lcc}
\hline Age group & No of patients & $\%$ total \\
\hline$<2$ years & 8 & $5 \cdot 8$ \\
2-10 years & 74 & 54 \\
10-14 years & 55 & $40 \cdot 2$ \\
\hline
\end{tabular}

Table 2 Indications for oral anticoagulation

\begin{tabular}{lcc}
\hline Diagnosis & No of patients & \% Total \\
\hline Deep venous thrombosis & 8 & $5 \cdot 8$ \\
Renal disease, such as & 7 & $5 \cdot 1$ \\
nephrotic syndrome & & \\
Prosthetic cardiac valve & 101 & $73 \cdot 7$ \\
Protein C deficiency & 4 & $2 \cdot 9$ \\
Antithrombin III deficiency & 1 & $0 \cdot 8$ \\
Other & 16 & 11.7 \\
\hline
\end{tabular}

Royal Manchester

\author{
Correspondence to: \\ Dr D I K Evans, Royal \\ Manchester Children's \\ Hospital, Hospital Road \\ Pendlebury, Manchester \\ Accepted for publication \\ 20 January 1992 \\ D I K Evans \\ Reference Laboratory \\ for Anticoagulant \\ Withington Hospital, \\ Manchester \\ Children's Hospital,
}


Table 3 Target levels for oral anticoagulation

\begin{tabular}{|c|c|c|c|}
\hline Diagnosis & $\begin{array}{l}\text { Mean lower limit } \\
\text { desired INR }\end{array}$ & $\begin{array}{l}\text { Mean upper limit } \\
\text { desired INR }\end{array}$ & No of replies \\
\hline $\begin{array}{l}\text { Prosthetic cardiac valve } \\
\text { Venous thrombosis (all causes) }\end{array}$ & $\begin{array}{l}2 \cdot 73 \text { (range } 2-3 \cdot 5) \\
2 \cdot 1 \text { (range } 2-3 \text { ) }\end{array}$ & $\begin{array}{l}4 \cdot 0 \text { (range } 3 \cdot 0-4 \cdot 5) \\
3 \cdot 25 \text { (range } 3-4 \cdot 5)\end{array}$ & $\begin{array}{r}29 \\
9\end{array}$ \\
\hline
\end{tabular}

were of erratic control in children under 2 years old.

QUESTIONNAIRE 2

Completed questionnaires were received from 11 of 22 centres dealing with paediatric cardiology or cardiothoracic surgery. All 11 centres provided information on their target levels for oral anticoagulation in children with prosthetic heart valves. Their responses can be summarised as follows: mean lower limit target INR 2.59 (range 2-3.5); mean upper limit target INR $3 \cdot 77$ (range 2.5-4.5). Again, there were no stated differences for the degree of anticoagulation in children under 2 years of age.

Five centres reported no problems with anticoagulation and four reported some erratic control, three poor compliance, and one excessive bruising.

Data on patient numbers from all 11 centres were not available, but six departments reported that over the past five years 68 children with prosthetic heart valves had been anticoagulated with warfarin. There had been two clinically relevant bleeding episodes and two thrombotic episodes.

\section{INITIATION OF ORAL ANTICOAGULATION IN} CHILDREN

Seven of $63(11 \cdot 11 \%)$ haematologists reported that they used a specific formula for the induction of oral anticoagulant treatment in children. The remainder used no stated formula, or their patients began oral anticoagulant treatment elsewhere, normally in a cardiothoracic centre. No consistent pattern for the initiation of treatment was reported: each used a different method. The stated doses ranged from 0.1 to $1.0 \mathrm{mgm} / \mathrm{kg} /$ day or were recorded as one quarter the adult dose, or one half the adult dose. Of the 11 cardiothoracic centres, only eight replied to the question, three used a formula, and five did not. Two used the same formula - that is, $200 \mu \mathrm{g} / \mathrm{kg}$ warfarin a day for two days, then $100 \mu \mathrm{g} / \mathrm{kg} /$ day for one day, subsequent doses being governed by the INR measured on day 4 .

TESTS USED FOR CONTROL OF ANTICOAGULANTS One hundred and eight $(\mathbf{7 8 \cdot 8 \%})$ children were controlled with a venous prothrombin time and $29(21 \cdot 2 \%)$ with a capillary test. Only a few children under the age of 2 were treated with anticoagulants; but of these, $50 \%$ were controlled with a capillary test.

\section{Discussion}

Most anticoagulant clinics supervised by haematologists do not treat children. Only three of the 62 reported in this study had seen five or more children in the previous five years.

The most common indication for anticoagulation in children of all ages is a prosthetic heart valve. The overall desired range of anticoagulation for these patients (INR $2 \cdot 7-4 \cdot 0$ ) is slightly less intense anticoagulation than the recommended BSH guideline values (INR 3.0-4.5) for adults with similar conditions. In the absence of a BSH guideline recommendation for oral anticoagulation in children it must be inferred that most clinicians based their anticoagulant control on their experience with adult patients. The overall optimal range of anticoagulation for children with prosthetic heart valves as reported by specialists in cardiac care was 2.6-3.8 INR.

A similar situation with established venous thrombosis (overall target therapeutic range $2 \cdot 1-3 \cdot 25)$ probably exists.

No single dosage regimen can be recommended for the induction of oral anticoagulant treatment in children. The replies were too few and too variable for a consensus to have become apparent.

The questionnaire survey indicates that most children with prosthetic cardiac valves and thrombotic disorders are treated with levels of anticoagulation similar to those currently recommended for adults with the same conditions. A recent survey of adults with mechanical prosthetic heart valves controlled with a rabbit brain thromboplastin (Simplastin) concluded that moderate intensity anticoagulation (prothrombin time ratio $1.5, \mathrm{NR}$ 2.65 ) was equally effective as high intensity anticoagulation (prothrombin time ratio 2.5 ; INR 9) but caused a significantly lower risk. ${ }^{2}$ The levels of anticoagulation achieved in our United Kingdom survey seem to be reasonable for safety and efficacy according to the present state of knowledge.

1 Poller L and BSH. British Society for Haematology Guidelines on oral anticoagulation: second edition. $\mathcal{Y}$ Clin Pathol 1990;43:177-83.

2 Saour JN, Sieck JO, Mamo LAR, Gallus AS. Trial of different intensities of anticoagulation in patients with
prosthetic heart valves. $N$ Engl $\Im$ Med 1990;322:428-32. 